\title{
Component neural systems for the creation of emotional memories during free viewing of a complex, real-world event
}

\section{Anne Botzung*, Kevin S. LaBar, Philip Kragel, Amanda Miles and David C. Rubin}

Department of Psychology and Neuroscience, Duke University, Durham, NC, USA

\section{Edited by:}

Tor Wager, Columbia University, USA

Reviewed by:

Elizabeth Kensinger, Boston College,

USA

Stephan Hamann, Emory University, USA

\section{${ }^{*}$ Correspondence:}

Anne Botzung, Duke University, Box 90086, 206 Psych/Soc, Durham, NC 27708-0086, USA.

e-mail: anne.botzung@duke.edu
To investigate the neural systems that contribute to the formation of complex, self-relevant emotional memories, dedicated fans of rival college basketball teams watched a competitive game while undergoing functional magnetic resonance imaging (fMRI). During a subsequent recognition memory task, participants were shown video clips depicting plays of the game, stemming either from previously-viewed game segments (targets) or from non-viewed portions of the same game (foils). After an old-new judgment, participants provided emotional valence and intensity ratings of the clips. A data driven approach was first used to decompose the $\mathrm{fMRI}$ signal acquired during free viewing of the game into spatially independent components. Correlations were then calculated between the identified components and post-scanning emotion ratings for successfully encoded targets. Two components were correlated with intensity ratings, including temporal lobe regions implicated in memory and emotional functions, such as the hippocampus and amygdala, as well as a midline fronto-cingulo-parietal network implicated in social cognition and self-relevant processing. These data were supported by a general linear model analysis, which revealed additional valence effects in fronto-striatal-insular regions when plays were divided into positive and negative events according to the fan's perspective. Overall, these findings contribute to our understanding of how emotional factors impact distributed neural systems to successfully encode dynamic, personally-relevant event sequences.

Keywords: declarative memory, affect, social cognition, sports psychology, functional neuroimaging, independent components analysis

\section{INTRODUCTION}

Exploring brain activity that supports memory encoding under natural conditions is particularly challenging. While asking human participants to retrieve real, complex events from their own personal life has become increasingly common in functional neuroimaging studies (see Svoboda et al., 2006; Cabeza and St Jacques, 2007 , for reviews), recording cerebral changes related to how these memories are initially formed has proven more difficult to examine experimentally. Hence, neural mechanisms supporting memory encoding have been almost exclusively studied through paradigms taken from laboratory settings, using lists of static items such as pictures or words (but see Hasson et al., 2008, for the use of a portion of a movie as the encoding phase condition). Even more challenging on both practical and ethical grounds is the task of investigating how the brain encodes memories that, in addition to their self-relevance and complexity, also vary in their emotional significance. Although progress has been made in elucidating amygdala-hippocampal interactions for the successful encoding of emotional stimuli (LaBar and Cabeza, 2006), it is unclear how these or other brain regions, such as those implicated in social

Abbreviations: DLPFC, dorso-lateral prefrontal cortex; fMRI, functional magnetic resonance imaging; GLM, general linear model; IC, independent component; ICA, independent component analysis; MTL, medial temporal lobe; NCAA, National Collegiate Athletic Association; PFC, prefrontal cortex; SPM, statistical parametric mapping; TPJ, temporo-parietal-occipital junction; UNC, University of North Carolina-Chapel Hill cognition (Van Overwalle, 2009) contribute to the encoding of dynamic, personally-relevant emotional episodes that take place within a social context.

A promising neuroimaging analysis technique that can be applied to address this problem is independent component analysis (ICA), which is a multivariate statistical approach that decomposes brain signals from temporally-extended event sequences into component parts. ICA is a data driven method that identifies and segregates distinct brain regions or networks based on their characteristic time course activity (Calhoun et al., 2001, 2003, 2009; Bartels and Zeki, 2004a,b, 2005). This method has proven to be useful for revealing functionally related brain regions during natural viewing of extended event sequences (e.g., watching movies), which, given their perceptual richness and complexity, are likely to engage a broad set of regions and connections (Jääskeläinen et al., 2008; Malinen et al., 2007; Calhoun et al., 2009). ICA differs from other multivariate analysis methods such as partial least squares (PLS, McIntosh et al., 1996) by decomposing signal into statistically independent rather than maximally variant sources. Additionally, ICA requires no a priori model of the data, which is particularly advantageous given the complexity of the stimuli at hand.

The present study applied this analytic approach to investigate the component neural systems that contribute to the formation of emotional memories that emerge in the context of a sporting event. As opposed to other temporally-extended event sequences such as movies, team sporting events contain individual plays, rendering it is easier to parse into shorter narrative structures with unique 
emotions. Research from sports psychology has shown that deeply committed fans experience sporting events vicariously - they are highly personally involved with the actions of the game; they selfidentify with team players; and they are prone to experience intense emotions related to the team's performance (Brown, 1986; Wann and Branscombe, 1993). Moreover, televised games include video footage and auditory overlay of crowd and commentator reactions as the game unfolds, which provides a social context despite viewing the game individually within the confines of an MRI scanner. From a research design perspective, another advantage of this paradigm is that the exact same play can be classified as either emotionally positive or negative according to the personal perspective of rival fans (plays that are emotionally positive for half the participants are emotionally negative for the other half). In this way, potential memory confounds such as differences in visual complexity or distinctiveness for events of opposing valence are completely controlled, which is difficult to achieve with other stimuli.

To achieve the study objectives, an archived college basketball game was selected and watched by dedicated fans of the two opposing teams while undergoing functional magnetic resonance imaging (fMRI). During scanning, a 35-min portion of a basketball game involving a traditional college basketball rivalry - Duke University vs. University of North Carolina - was freely viewed by fans from the two teams. Recognition memory was assessed post scanning, using video clips that depicted individual plays of the game. After judging whether they had previously viewed the play, participants rated the emotional intensity and valence of the clip.

The primary goal of this work was to improve our understanding of the brain networks involved in the formation of real-life emotional events, encoded under more ecological conditions, by (i) taking advantage of the data driven approach provided by ICA to identify component neural systems, and then, (ii) performing correlations between the neural activity identified with ICA and the emotional ratings for plays that were successfully encoded. This approach was taken over other measures of functional connectivity such region of interest (ROI) or seed based correlations in order to minimize spatial and temporal selection bias. As opposed to blind data analysis methods such as ICA, the more conventional general linear model (GLM) approach is hypothesis driven, i.e., it implies some prior knowledge about the shape of the expected hemodynamic response, in the context of a predictive model. Our second objective was then to use the ICA data we obtained as a guide for a GLM analysis, especially regarding the temporal characteristics associated with the exciting plays (i.e., peak of the related time courses), with the aim of building up a predictive model to be used with statistical parametric mapping.

\section{MATERIALS AND METHODS PARTICIPANTS}

Twenty college basketball fans of opposing teams, with no prior history of neurological or psychiatric impairment, participated in the study. Ten subjects were fans of the Duke University Men's Basketball team [mean age $=20.30$ years, standard deviation $(\mathrm{SD})=3.68$ ], and 10 subjects were fans of the rival team [the University of North Carolina-Chapel Hill (UNC), mean age $=22.70$ years, $\mathrm{SD}=2.94$ ). All participants were recruited through a set of questionnaires aimed at determining their level of fandom. The first two questionnaires involved self-ratings about: (i) their level of basketball fandom and their fan-related behavior with regard to their favorite men's college basketball team (adapted from Wann et al., 2003), (ii) their own and others' perceptions of them as fans, as well as their feelings and behaviors associated with their fandom (adapted from Wann and Branscombe, 1993). Three additional questionnaires were used to determine the participants' level of knowledge about basketball in general, National Collegiate Athletic Association (NCAA) men's basketball, and their favorite men's college basketball team (Duke or UNC). These questionnaires were constructed for the purpose of this study and piloted on 21 participants to select the most appropriate questions. The participants had to satisfy stringent criteria to qualify: they must have ranked themselves as having a high level of fandom, and maintained an overall average of $75 \%$ correct responses on the basketball knowledge questionnaires. Based on pilot data showing low level memory performance in females, only males were recruited to participate to the present study. All participants gave informed written consent prior to their inclusion in the study, which was approved by the Institutional Review Board at Duke University Medical Center.

\section{PROCEDURE AND STIMULUS MATERIALS}

Participants freely watched a portion of a basketball game in the scanner, and their recognition memory for the most eventful subsections was tested afterwards. We selected a basketball game between Duke and UNC, played at UNC in 2001, based on its competitiveness and variability in emotional intensity. None of the participants were familiar with this particular game. The participants were presented with a 35-min section from the middle of the game. Within this portion, 40 emotionally intense short segments were identified for subsequent statistical analysis. Each segment was between 10 and $24 \mathrm{~s}$ in length (average $=16.33 \mathrm{~s} ; \mathrm{SD}=4.47 \mathrm{~s}$ ) and portrayed a play characterized by an exciting result such as a shot (made or missed), a difficult pass, or a good defensive play. Segments were divided according to the fan's perspective into those that were emotionally positive $(n=20)$ and emotionally negative $(n=20)$. For instance, a segment showing a shot made by a player from the fan's team corresponded to a stimulus that was emotionally positive for the fan's team, but emotionally negative for the fan of the rival team. The viewing session was divided into four runs of approximately equal duration. The subjects were instructed to enjoy and attend to the game closely in preparation for an impending memory test (intentional encoding).

Thirty minutes after the viewing session, participants completed a recognition task outside the scanner. During the recognition task, subjects were shown 80 clips from the basketball game. The clips were either viewed by the subjects in the scanner ( 40 old items), or were from portions of the game that were not viewed (40 new items). The new segments were matched to the old ones in valence (20 positive and 20 negative plays according to one fan's perspective) and in duration (average $=15.58 \mathrm{~s} ; \mathrm{SD}=3.37 \mathrm{~s}$ ). The fans were shown the clips in random order. Game time and score were covered during the task. Based on pilot studies, this design ensured recognition memory performance in a range of $80-85 \%$ accuracy. Presentation of each clip was followed by a 6-s fixation during which participants were asked to make an old/new judgment (yes/ no response). The memory judgment was followed by a confidence 
screen that appeared for $4.5 \mathrm{~s}$, instructing the participants to rate how confident they were in their decision on an eight-point scale (from 1 = "very low" to $8=$ "very high"). Then, an emotional rating screen appeared for $4.5 \mathrm{~s}$, instructing the participants to rate each clip for valence and intensity on a 4-point scale (from -1 up to -4 for negative events; or from 1 up to 4 for positive events), according to the emotion felt while viewing these sequences in the scanner. These ratings were collected at the time of recognition memory testing so as not to interrupt the free viewing period. The emotional rating was followed by short periods of fixation between trials (mean duration $=3 \mathrm{~s}$, range $=2-5 \mathrm{~s}$ ).

\section{fMRI METHODS}

\section{Scanning}

Images were collected using a 4-T GE scanner. Video was presented using liquid crystal display goggles (Resonance Technology, Northridge, CA, USA), and sound was diffused through audio headphones. Cogent 2000 (Wellcome Department of Cognitive Neurology, London, UK) was used for the presentation and timing of runs. Scanner noise was reduced with earplugs, and head motion was minimized using foam pads and a headband. Anatomical scanning started with a T2-weighted sagittal localizer series. The anterior commissure and posterior commissure were identified in the midsagittal slice, and 34 contiguous oblique slices were prescribed parallel to the AC-PC plane. High-resolution T1-weighted structural images were collected first, with a 12.3-ms repetition time (TR), a 5.4-ms echo time (TE), a 24-cm field of view (FOV), a $256^{2}$ matrix, 68 slices, and a slice thickness of $1.9 \mathrm{~mm}$. Functional images were subsequently acquired using an inverse spiral sequence with a 1.5-s TR, a 31-ms TE, a $24-\mathrm{cm}$ FOV, a $64^{2}$ matrix, and a $60^{\circ} \mathrm{flip}$ angle. Thirty-four contiguous slices were acquired with the same slice prescription as the anatomical images. Slice thickness was $3.8 \mathrm{~mm}$, resulting in $3.75 \mathrm{~mm} \times 3.75 \mathrm{~mm} \times 3.8 \mathrm{~mm}$ voxels.

\section{fMRI analyses}

Preprocessing and statistical analyses were conducted using SPM5 software (Wellcome Department of Cognitive Neurology, London, UK), implemented in MATLAB (2007b, The MathWorks Natick, MA, USA). Functional images were slice time corrected, realigned to correct for motion artifacts, spatially normalized to the Montreal Neurological Institute (MNI) template, and then spatially smoothed using an 8-mm full-width-half maximum isotropic Gaussian kernel. The subsequent analyses were carried out using both SPM5 and group-ICA toolbox GIFT (http://icatb.sourceforge.net/) (Calhoun et al., 2001, 2003, 2009).

For ICA, 20 spatially independent components (ICs) were first determined using Infomax algorithms (Bell and Sejnowski, 1995) implemented in the GIFT toolbox. Components related to the 35 min of the basketball game were first calculated for the group, and individual subject components were created using back reconstruction. T maps were created by performing a random effects analysis using individual subjects' component maps as input, using a threshold of $p<0.001$ and a minimum extent threshold $(k)$ of five voxels. In order to select meaningful ICs, we used a systematic anatomical criterion that corresponded to a minimum of $85 \%$ of total gray matter in the spatial pattern of activity associated with each component. Based on visual inspection of their spatial distribution on the group T maps, activity related to five ICs were discarded because they reflected motion artifacts located in the ventricles and/or at the edge of the brain. To check the validity of this anatomical criterion, we interrogated the amplitude of the components' time courses averaged for the previously identified segments. Low amplitude was generally observed for patterns including less than $85 \%$ gray matter. Using this procedure, nine functional ICs were identified and subjected to further analyses. Time courses associated with the successfully encoded plays were first obtained for each component using windowed averaging time-locked to the end of the play. Time courses were obtained around that point, from 10 TRs before to 8 TRs afterwards (total length $=18$ TRs). Withinsubject correlations were then calculated between the maximum component time-course values [from the reference point to $7.5 \mathrm{~s}$ after (5 TRs)] and post-scanning emotion ratings binned according to memory performance. This time window was chosen based on where one would reasonably expect a hemodynamic response; that is, at end of the play where the emotional impact is revealed (ball was stolen, shot went into the basket, etc.). Subjects' correlation statistics were converted to $Z$-scores for making group inferences.

As for the subsequent SPM analysis, trials were created based on successful encoding (correct or incorrect in the subsequent recognition task), intensity ratings (low, medium, or high) and valence ratings (positive or negative). Statistical parametric maps were created using contrasts of these conditions (i.e., correct high intensity trials vs. correct low intensity trials, or correct positive trials vs. correct negative trials) in the context of the GLM. Because memory performance was high, only correct memory trials were included in the SPM analyses, and a formal comparison of hits vs. misses in memory was not feasible. Based on the shape of the time-courses we obtained from the ICA analysis, evoked brain responses to successful encoding was modeled with a box-car function convolved with a hemodynamic response function for two TRs around the end of the play. The intensity ratings were considered in absolute values, and to homogenize the number of trials per intensity ranking, they were rescaled as follows: low $=1$; medium $=2$; high $=3-4$. To take into account the fact that positive segments were rated as more intense than negative segments (see Results), intensity was modeled as a covariate of non-interest in the context of the valence comparison. Individual correct high vs. low intensity and correct positive vs. negative contrast images were subjected to random effect analyses (one-sample $t$ test). In order to test the valence by intensity interaction, an analysis of variance (ANOVA) was conducted using a full factorial design implemented in SPM, with intensity (high vs. low) and valence (positive vs. negative) as factors. This allowed us to identify which regions were responsible for the difference in intensity in the successful encoding of positive segments compared to negative ones. For all GLM analyses, the significance threshold was set at $p<0.001$, with a minimum extent threshold of five contiguously activated voxels (uncorrected). The significance threshold was set at $p<0.005$ (uncorrected, $k=3$ ) to explore regions of the MTL.

\section{RESULTS}

\section{BEHAVIORAL DATA}

The overall proportion of successfully recognized old and new video clips was 0.86 (see Table 1 ). The hit rate was 0.83 , the false alarm rate was 0.11 , and the resulting $d^{\prime}$ was equal to 2.18 , indicating a good level of discrimination between old and new items. Considering old 
items only (the 20 positive and 20 negative segments used for the fMRI analysis), the independent $t$-tests performed at the subject level indicated no difference between participant groups (Duke vs. UNC fans) neither in accuracy $\left(t_{20}=0.05, p=0.96\right)$, confidence $\left(t_{20}=1.02\right.$, $p=0.32)$ or emotional intensity $\left(t_{20}=0.50, p=0.62\right)$, nor in the rating reaction times (RTs) (confidence: $t_{20}=1.23, p=0.23$; emotional intensity: $\left.t_{20}=1.48, p=0.16\right)$. Duke fans were faster to make responses in the recognition memory task than UNC fans $\left(t_{20}=2.32\right.$, $p=0.03)$. As for the effect of valence, the paired $t$-test performed at the subject level indicated no significant difference between positive and negative items neither in accuracy $\left(t_{20}=1.68, p=0.11\right)$, nor in confidence $\left(t_{20}=0.46, p=0.65\right)$, but positive items were judged as being more intense than the negative ones $\left(t_{20}=2.22, p=0.04\right)$.

\section{fMRI DATA \\ ICA results}

Individual components. As shown in Figure 1, we isolated a "visual IC," which included areas related to visual stimulation (occipital cortex, Figure 1A), as well as an "auditory IC," which consisted of areas related to auditory stimulation (lateral superior temporal cortex bilaterally, Figure 1B). There was also a "sensorimotor IC," which included regions of the sensory-motor system (superior fronto-parietal network and cerebellum bilaterally, Figure 1C). The "dorsal fronto-parietal IC" corresponded to the dorsal attention

Table 1 | Mean (SD) behavioral data and reaction times (RTs, in $\mathrm{ms}$ ) for recognition, confidence, and intensity judgments for the old items as a function of self-identified fan group $(*$ indicates significant group difference, $p=0.03$ ).

\begin{tabular}{lrrr}
\hline & Duke fans & UNC fans & All subjects \\
\hline Recognition & $83 \%(0.11)$ & $83 \%(0.11)$ & $83 \%(0.11)$ \\
Recognition RT & $1018(302)^{*}$ & $1445(497)$ & $1231(456)$ \\
Confidence & $7.01(0.52)$ & $6.72(0.76)$ & $6.87(0.65)$ \\
Confidence RT & $1004(193)$ & $1199(462)$ & $1102(360)$ \\
Intensity & $2.07(0.42)$ & $2.19(0.66)$ & $2.13(0.54)$ \\
Intensity RT & $1411(287)$ & $1688(518)$ & $1549(432)$
\end{tabular}

network (anterior cingulate cortex and fronto-parietal regions, Figure 1D). The fifth IC was almost exclusively left lateralized and included regions that are involved in language and semantic processing, such as the infero-lateral prefrontal regions, as well as lateral temporal areas extending through the left temporo-parietal-occipital (TPO) junction ("left fronto-temporo-parietal IC," Figure 1E). An analogous contralateral pattern was observed for the sixth "right fronto-temporo-parietal IC," with additional areas in left homologue regions as well as in medial prefrontal and posterior cingulate cortices (Figure 1F). The seventh IC included regions of the self/social cognition/default network ("default network IC1"), such as medial frontal cortex, and a large posterior volume comprising the cingulate cortex, cuneus, and precuneus medially, and extending bilaterally through the TPO junction (Figure 1G). The eighth IC ("default network IC2") corresponded to similar midline regions, but with activity more concentrated in frontal structures extending into infero-lateral parts of the prefrontal cortex (PFC), as well as lateral and medial temporal lobe cortices bilaterally (see Figure 2A; Table 2). Finally, the last identified IC ("MTL-visual IC") included medial temporal and frontal regions and extended posteriorly into visual areas in a continuous strip from the amygdala to the ventral occipitotemporal cortices via the hippocampus and parahippocampal and retrosplenial cortices (Figure 2B; Table 2).

Correlations between ICs and post-viewing ratings. Correlations were performed between peak component activity and emotional ratings (see Materials and Methods for details). The only significant correlations were between intensity ratings and both the default network IC2 and the MTL-visual IC ( $p=0.0016$ and 0.0015 , respectively). Comparing the time courses of these two ICs, it is interesting to note that the response associated with default network IC2 peaked earlier (by approximately $2 \mathrm{~s}$ ) than the response associated with the MTL-visual IC (see Figure 2B).

\section{Comparisons in the GLM}

Intensity. In support of the ICA results, comparing successfully encoded high vs. low intensity segments revealed activity in medial superior and anterior PFC, in inferior frontal gyri bilaterally, in

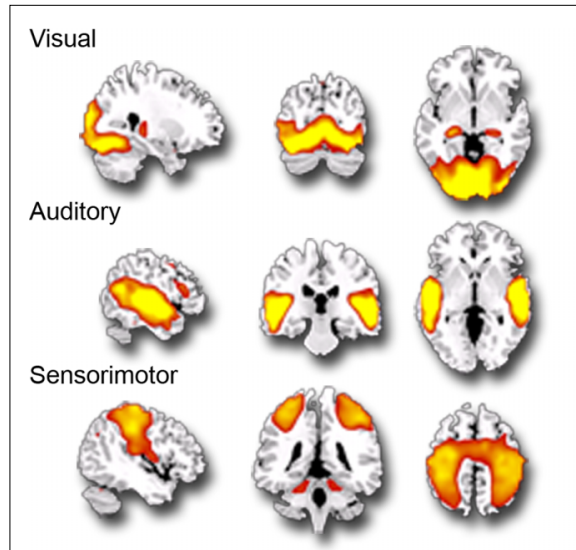

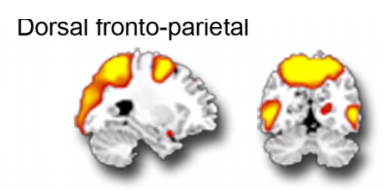

Left fronto-temporo-parietal

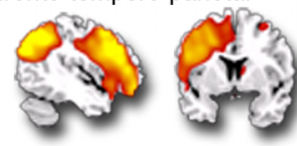

Right fronto-temporo-parietal
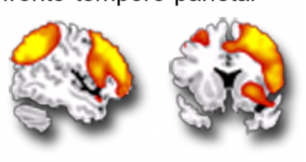
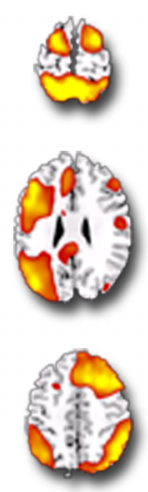

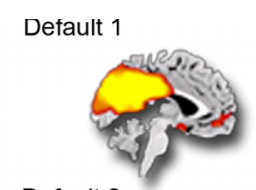

Default 2
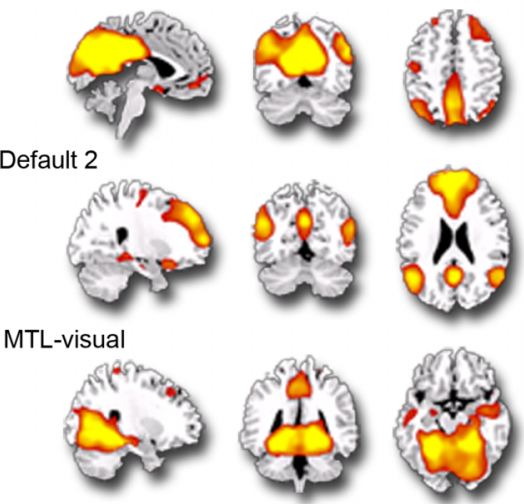

FIGURE 1 | Independent components (ICs) identified during the free viewing of the 35-min portion of the basketball game ( $p=0.001$; $k=5$ ). This figure represents the 9 IC spatial maps identified, each overlaid on the sagittal, coronal and axial planes of an anatomical template. 


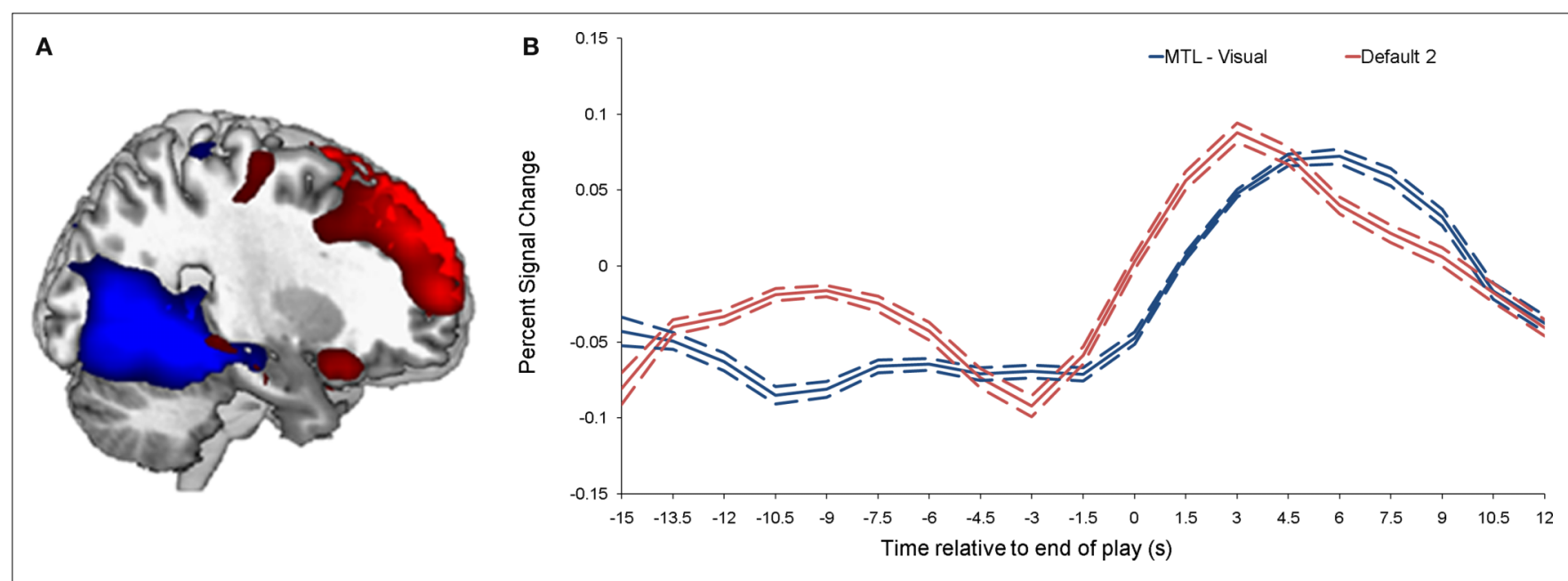

FIGURE 2 | Brain activity related to the two ICs that correlated with emotional intensity ratings. (A) Shows the "MTL-visual IC" (blue) and the "default network IC2" (red), and their corresponding time-courses are shown in (B). The dotted lines indicate the standard deviation.

the posterior cingulate cortex, in posterior parahippocampal and lingual gyri bilaterally, and in a cluster including the right anterior hippocampus and the amygdala (Table 3 ). The reverse comparison did not yield any significant results.

Valence. Comparing successfully encoded positive and negative segments from the fan's perspective revealed valence effects that were not found in the ICA analysis. Greater activity associated with positive segments was observed in regions of the dorso-lateral PFC (DLPFC) bilaterally (right BA 9 and left BA 6), a cluster including the left insula and superior temporal and inferior frontal gyri (BA 38, 47, 13), as well as the right precuneus (BA 7) (Table 3). Successfully encoded negative segments, by comparison with positive ones, elicited small loci of enhanced activity in the right thalamus, the left striatum, the right pre- and post-central gyri (BA 3, 5, 6), and posteriorly in the left insula (BA 13) (Table 3).

Intensity by valence interaction. A greater difference in activity between high and low intensity for successfully encoded positive relative to negative segments was observed in the right amygdala, the right striatum (caudate) and the left orbito-frontal cortex (BA 47) (Table 3; Figure 3). We did not observe any reverse interaction effect.

\section{DISCUSSION}

In the context of a social sporting event, we demonstrated, using a data-driven analysis, a modulatory effect of intensity on the neural bases supporting emotional memory formation under free viewing conditions. Two ICs correlated with emotional intensity ratings - the MTL-visual IC, which comprised key regions of declarative memory, emotion, and visual perception circuitries, and the default network IC2, which included midline regions of the typical default network, related PFC areas involved in self-referential processing and social cognition, plus the hippocampus. Conventional GLM analyses were then performed on the basis of the temporal characteristics of these two ICs. These latter results supported those obtained with ICA but revealed additional valence effects and valence $\times$ intensity interactions. These results are discussed, in turn, below.

\section{IC - EMOTIONAL RATING CORRELATIONS}

Both the default network IC2 and the MTL-visual IC correlated with the emotional intensity ratings of plays that were correctly recognized in a subsequent memory test. It is first worth noting that successful encoding of emotional information in a more ecologically valid setting appears to recruit a much larger set of brain regions than what is usually observed using list learning paradigms, which have typically revealed regions of the MTL and inferior PFC areas (Dolcos and Denkova, 2008). The main findings from these latter studies is enhanced memory for emotional information that benefits from (i) the joint contribution from both MTL- and PFCbased mechanisms, and (ii) the critical role of emotional intensity, through the involvement of the amygdala, in mediating these effects. For instance, a stronger correlation was observed between MTL and amygdala during the encoding of emotional than neutral stimuli (Dolcos et al., 2004), an effect that seems to persist over time (Ritchey et al., 2008). Positive correlations were also found between different parts of lateral PFC and the amygdala, suggesting the role of the PFC in the formation of emotional memories by enhancing strategic, semantic and working memory processes (Dolcos et al., 2004; LaBar and Cabeza, 2006). These seed based connectivity findings show which regions covary with the MTL during encoding of emotional stimuli. The current ICA approach revealed one functionally connected network containing many of these regions.

Implication of both the amygdala and the hippocampus has also been shown in the context of natural viewing. Activity in both structures was revealed in fMRI using a GLM approach during appreciation of humor moments (while participants were passively viewing full-length episode of a sitcom, Moran et al., 2004), whereas direct influence of the amygdala on parahippocampal cortex and ventrolateral PFC was reported in a PET study using structural 
Table 2 | Independent components analysis (ICA) results during free viewing that positively correlated with behavioral ratings of emotional intensity.

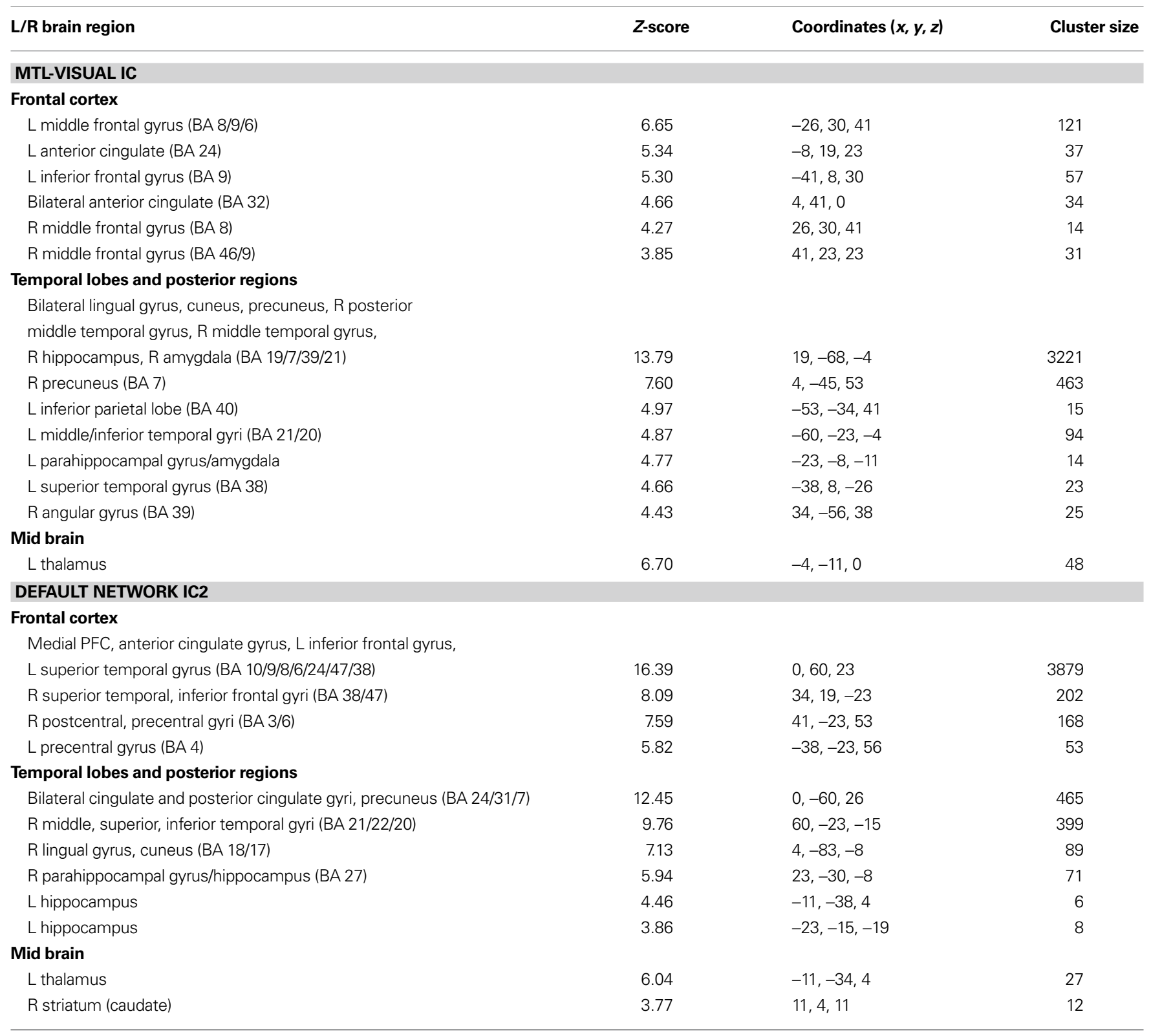

One component included medial temporal and ventral visual areas associated with memory, emotion, and complex visual perception (MTL-visual; see Figure 1) while the second component included brain regions involved in self-referential/default mode processing, social cognition, and memory (default network IC2; see Figure 1). Coordinates are in Montreal Neurological Institute stereotaxic space.

$B A$, Brodmann area; $L$, left hemisphere; PFC, prefrontal cortex; $R$, right hemisphere.

equation modeling (Kilpatrick and Cahill, 2003), as sustaining the encoding of negative vs. neutral emotionally arousing film clips. Consistent with these findings, we observed in the MTL-visual IC, for both positive and negative segments, activity in different lateral parts of the PFC bilaterally and in the MTL, including the hippocampus and the amygdala bilaterally. This IC also shows extended activity in posterior ventral occipitotemporal cortices, which suggests that memory formation for complex, real-life stimuli requires the involvement of a coupling among the hippocampus, amygdala and posterior sensory regions at encoding.
In addition to this IC, the default network IC2 comprised regions typically implicated in social cognitive functions and processing of self-related stimuli, such as during autobiographical memory retrieval (Addis et al., 2004; Cabeza and St Jacques, 2007; Botzung et al., 2008; Buckner et al., 2008). Hasson et al. (2008) combined natural viewing and a data-driven method (inter-subject correlations) to identify brain regions whose activity was correlated across subjects for portions of a movie that were successfully encoded. Based on the enhanced activity the authors observed in midline structures, TPJ and temporal 
Table 3 | Results from the general linear model analysis.

\begin{tabular}{|c|c|c|c|}
\hline L/R brain region & Z-score & Coordinates $(x, y, z)$ & Cluster size \\
\hline \multicolumn{4}{|l|}{ HIGH VS. LOW INTENSE PLAYS } \\
\hline \multicolumn{4}{|l|}{ Frontal cortex } \\
\hline R inferior frontal PFC (BA 46) & 3.49 & $45,38,4$ & 21 \\
\hline Anterior and superior medial PFC (BA 8/9) & 3.43 & $0,41,49$ & 73 \\
\hline L inferior PFC (BA 47/45) & 3.36 & $-49,26,0$ & 13 \\
\hline R parahippocampal gyrus/lingual gyrus (BA 19) & 3.91 & $19,-56,-4$ & 181 \\
\hline L temporo-parietal junction (BA 39/40) & 3.79 & $-49,-68,41$ & 145 \\
\hline \multicolumn{4}{|l|}{ Temporal lobes } \\
\hline L inferior temporal gyrus (BA 20) & 3.12 & $-60,-34,-15$ & 5 \\
\hline R hippocampus/amygdala* & 2.79 & $34,-8,-15$ & 4 \\
\hline R precuneus (BA 7) & 3.08 & $8,-34,45$ & 5 \\
\hline \multicolumn{4}{|l|}{ NEGATIVE VS. POSITIVE PLAYS } \\
\hline $\mathrm{R}$ thalamus & 3.31 & $19,-23,0$ & 6 \\
\hline L striatum (caudate) & 3.25 & $-23,-34,11$ & 38 \\
\hline R postcentral gyrus/parietal lobe (BA 5) & 3.17 & $26,-45,68$ & 6 \\
\hline L posterior insula (BA 13) & 3.13 & $-34,-26,19$ & 5 \\
\hline R postcentral and precentral gyri (BA 3/6) & 3.10 & $49,-15,53$ & 30 \\
\hline \multicolumn{4}{|l|}{ VALENCE (POSITIVE VS. NEGATIVE) BY INTENSITY INTERACTION } \\
\hline R striatum (caudate) & 3.49 & $4,15,4$ & 21 \\
\hline R amygdala & 3.19 & $15,-4,-19$ & 14 \\
\hline
\end{tabular}

Main effects of arousal, valence, and their interaction are indicated. Coordinates are in Montreal Neurologic Institute stereotaxic space. $B A$, Brodmann area; $L$, left hemisphere; $P F C$, prefrontal cortex; $R$, right hemisphere. ${ }^{*} p<0.005$.

A

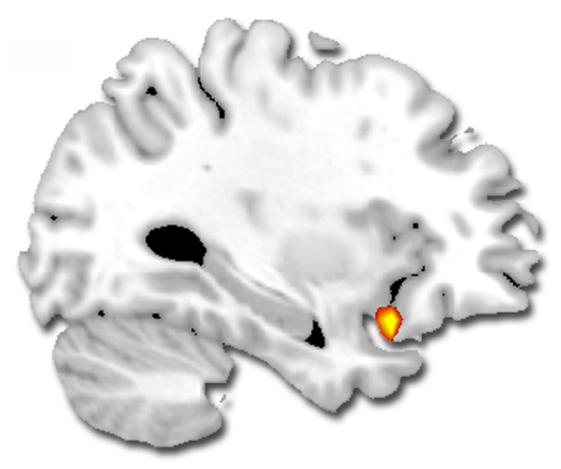

B

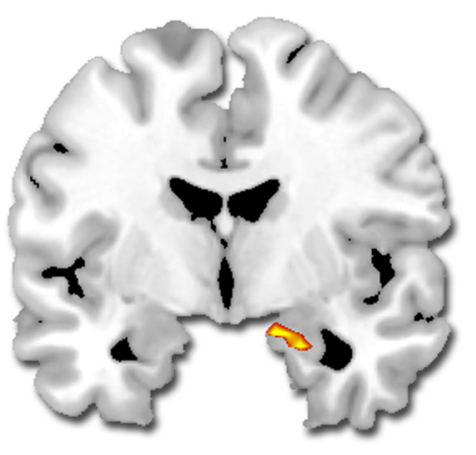

C

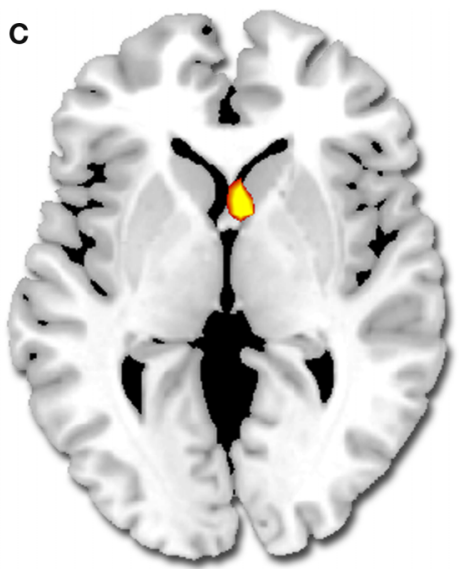

FIGURE 3 | Regions showing a greater difference in activity for successfully encoded positive relative to negative segments $(p<0.001, k=5)$ : (A) left orbito-frontal cortex, (B) right amygdala, (C) right striatum.

poles, Hasson et al. (2008) suggested that modulation of social cognitive processes can impact episodic memory formation for real-time human interactions. Our data extend this idea by using
ICA rather than inter-subject correlations and by showing that successful encoding activity in these regions further correlates with ratings of emotional intensity. Moreover, the present study 
uses a sporting event viewing paradigm that permits the encoding of memories in a close-to-real-life socioemotional setting that has direct relevance to the participants as highly identified fans. Further research is warranted to determine whether emotionenhanced activity in these midline cortical regions reflects selfrelevant processing, processing of social context, or processing of the interpersonal aspects of the player actions.

Inspection of the time courses associated with these two intensitycorrelated ICs revealed that the default network IC2 temporally preceded the MTL-visual IC by approximately $2 \mathrm{~s}$. This finding may suggest that processing self-relevant and social information (through the interaction between the hippocampus and the selfsocial cognition network) might be predictive (as a preliminary step) of the subsequent interaction between the hippocampus, the amygdala, and posterior cortical regions. Of particular interest is the enhanced activity seen in these posterior regions, including temporal, parietal and occipital cortices, which are associated with the recall of sensory features of real-life stimuli, such as autobiographical memories (Cabeza and St Jacques, 2007; Botzung et al., 2008). In both ICs, activity in the hippocampus was observed bilaterally, but it was more anteriorly extended in the MTL-visual IC to encompass the left head region, which is more reciprocally connected to the amygdala. This result might suggest that the hippocampus acts as a bridge between social cognitive processes and affective-perceptual processes to bind together these aspects of the memory during initial encoding.

\section{OTHER ICS RELATED TO NATURAL VIEWING OF A BASKETBALL GAME}

Seven additional ICs were identified during game viewing that involved functionally meaningful brain activation (i.e., were contained within gray matter and were not related to motion artifacts). Only a few studies have combined the use of natural viewing and ICA (Malinen et al., 2007; Jääskeläinen et al., 2008), some of which focused specifically on how visual features of the movie related to visual functional subdivisions (Bartels and Zeki, 2004a,b, 2005). Consistent with this literature, and as expected in the context of a continuous video sequence containing multimodal stimuli, the visual and auditory ICs corresponded to the visual and the auditory systems (Bartels and Zeki, 2005; Malinen et al., 2007; Jääskeläinen et al., 2008). In previous studies, though, several visual ICs were isolated, corresponding to finer functional subdivisions within the visual cortex, which is likely to be accounted for by a greater number of ICs initially specified (Bartels and Zeki, 2005). Also reported in the literature and replicated here is the left-lateralized frontotemporo-parietal IC, including the infero-lateral PFC and lateral temporal cortex extending into TPJ (Bartels and Zeki, 2005), which given the region's location could be related to language processing. In the present study, a similar IC was also observed but focused in the right hemisphere (right fronto-temporo-parietal IC). Given the known involvement of the right hemisphere for spatial processing, the right fronto-temporo-parietal IC might be related to this function or, alternatively, to right-hemispheric communicative functions (processing of affective prosody, etc.).

A sensorimotor IC was reported in one of the aforementioned studies, but was related to the tactile stimulus blocks the authors introduced during the viewing session (Malinen et al., 2007). In our case, since no tactile stimulation was given and no motor response was required, the sensory-motor IC we isolated might reflect the involvement of sensory-motor representations elicited by observing the movements and actions of the players (see Aziz-Zadeh and Ivry, 2009, for a recent review). An IC similar to the one we identified as corresponding to the canonical dorsal attention network (anterior cingulate cortex and dorsal fronto-parietal regions) has been previously reported in context of a working memory task (Wolf et al., 2008), and previous studies have shown similarities in brain activation patterns mediating spatial attention and working memory (LaBar et al., 1999). Similar to some previous ICA studies (Damoiseaux et al., 2006; Esposito et al., 2006; Golland et al., 2007; Malinen et al., 2007), we observed two distinct ICs that both included medially located cortical structures (amPFC, PCC, precuneus) and the TPJ. These regions are core components of the so-called "default-network" and they are also known to support self-referential processing and other aspects of social cognition (Van Overwalle, 2009). One of these networks (default network IC1) was characterized by more extended activity in posterior midline structures but less extended in the medial PFC, whereas the second one (default network IC2) uniquely included the inferolateral PFC and lateral and medial temporal cortices bilaterally (as described above).

\section{ICA VS. GLM RESULTS: INTENSITY AND VALENCE EFFECTS}

Consistent with the known temporal lag of the hemodynamic response, ICs that were positively correlated with emotional intensity ratings peaked approximately 3-6 s after the end of the tagged segments (the portions of the ongoing game where the emotional impact was maximal). This temporal information was then used to inform the construction of a GLM-based model investigating the effects of valence and intensity (and their interaction) on successfully encoded memories. For the intensity analysis, both approaches elicited similar results, with additional regions revealed by ICA, especially homologue regions already active in the opposite hemisphere. For instance, similar to the MTL-visual IC, GLM revealed intensity-related activity in the right dorso-lateral PFC, in the right amygdala and hippocampus, and in the parahippocampal gyri extending to lingual gyri bilaterally; in contrast, the left amygdala, left dorsolateral PFC, and anterior hippocampus only emerged in the ICA analysis. The ICA analysis also revealed activity in posterior regions that was more widespread, extending to the precuneus. Similarly, as opposed to the default network IC2, GLM revealed intensity-related activity in the left (but not right) TPJ, and activity in midline structures was less extended. These results are generally in accordance with other method comparison studies, which showed that patterns of activity revealed by ICA tend to be spatially more extensive (Calhoun et al., 2001; Bartels and Zeki, 2004a; Hasson et al., 2008; Malinen et al., 2007). Compared to viewing simple discrete stimuli, natural viewing conditions tend to elicit ICs comprising homologous areas in contralateral regions, probably reflecting greater inter-hemispheric reliance to support information processing during complex multisensory stimulation (Bartels and Zeki, 2005).

In contrast to the methodologically consistent results for emotional intensity, significant effects of valence emerged only in the GLM analysis. This discrepancy suggests that intensity is more critical than valence for defining spatially independent networks 
during emotional memory formation. It is possible that valencebased ICs would be found if the ICA analysis were performed on a subspace containing a greater number of dimensions; however, as the number of dimensions increases, ICA estimation becomes less stable (Li et al., 2007). Nonetheless, the GLM analysis shows that individual brain areas are sensitive to valence, even if this affective dimension is not as important for segregating spatially discrete networks in the functional time series.

Behaviorally, positively valenced plays (plays that benefited the fan's team) were rated as more emotionally intense than negative plays. After controlling for this intensity bias, positivelyvalent plays recruited more activity than negatively-valent plays in bilateral dorsal frontal and left peri-insular regions whereas negatively-valent plays recruited more activity in left posterior insula and caudate, and right thalamus and somatosensory cortices. The anterior insula and overlying frontal convexity serves as a convergence point for representations of bodily states with social and contextual information to provide a neural substrate of the conscious experience of our own emotions (Craig, 2002) as well as the empathic feeling of others' emotions (Adolphs et al., 1995). The DLPFC is thought to support organization and associative encoding (for reviews, see Simons and Spiers, 2003; Blumenfeld and Ranganath, 2007). These processes may have been amplified during the successful encoding of pleasant social memories, consistent with psychological theories of attentional broadening and contextual elaboration during positive states (Fredrickson, 2001). As for negative plays, enhanced activity in the thalamus, posterior insula and somatosensory cortex might reflect greater visceralsensory feedback. Altogether, these findings further extend laboratory studies indicating that emotionally positive and negative information seem to be processed in different ways during their initial encoding (Dolcos et al., 2004; Kensinger and Schacter, 2008; Mickley and Kensinger, 2008).

Finally, the right amygdala, right caudate, and left ventrolateral PFC showed an enhanced sensitivity to emotional intensity in the context of positively-valent plays (intensity $\times$ valence interaction). The caudate is implicated in social learning (Balleine et al., 2007; Delgado, 2007) and the amygdala and ventrolateral PFC predict subsequent memory for emotional stimuli (Dolcos et al., 2004; Kensinger and Schacter, 2008). Functional imaging studies have shown that overall the amygdala is sensitive to emotional arousal, while also showing either preference for negative valence, or a valence-independent effect (Dolcos and Denkova, 2008). Importantly, our result challenges the traditional view linking the amygdala preferentially to negative emotions (Adolphs et al., 1995; Zald, 2003). Hence, the GLM results indicate a better sensitivity of the amygdala for encoding arousal in the context of positively-valent events when the setting is a personallyrelevant and enjoyable social experience.

\section{REFERENCES}

Addis, D. R., Moscovitch, M., Crawley, A. P., and McAndrews, M. P. (2004). Recollective qualities modulate hippocampal activation during autobiographical memory retrieval. Hippocampus 14, 752-762.

Adolphs, R., Tranel, D., Damasio, H., and Damasio, A. R. (1995). Fear and the human amygdala. J. Neurosci. 9, 5879-5891.

Aziz-Zadeh, L., and Ivry, R. B. (2009). The human mirror neuron system and embodied representations. $A d v$. Exp. Med. Biol. 629, 355-376.

Balleine, B. W., Delgado, M. R., and Hikosaka, O. (2007). The role of the dorsal striatum in reward and

\section{POTENTIAL LIMITATION}

Our objective was to examine emotional encoding in conditions that would be as natural, complex, close as possible to real-life, which implies more compromises than in the context of laboratory settings. In order to conceive a memory task that would enable us to control for successful encoding, we identified as many exciting segments as we could that could be matched both in terms of valence and duration. This recognition task was administered after a 30-min delay, which is fairly standard in the context of recognition memory assessment (see for instance Warrington, 1984, 1996). Selecting more exciting segments was not possible within the same game, unfortunately, due to the limited total number of plays in a game. As a consequence, we could only include in the analyses the segments that were remembered and did not have enough forgotten events to perform a "hits vs. misses" comparison. Future studies should attempt a remembered vs. forgotten analysis, which would confirm that the effects we observed are undeniably tied to emotional memory encoding.

\section{CONCLUSION}

Natural viewing of a basketball game in dedicated fans from the opposing teams was used to elicit rapid shifts in positively and negatively valenced emotional responses during encoding. Not only do the fMRI results confirm prior findings of arousalmediated memory functions of the amygdala and hippocampus, but they also implicate an extended visual network, as well as a social cognitive network, which are not typically associated with emotional recall using typical laboratory stimuli. Overall, the use of a sports game paradigm in highly engaged fans combined with data-driven ICA methodology provides novel insights into the brain systems involved in forming emotional memories during complex social events.

\section{AUTHOR CONTRIBUTIONS}

Anne Botzung, Kevin S. LaBar and David C. Rubin conceived and designed the experiment. Anne Botzung and Amanda Miles performed the experiment. Anne Botzung and Philip Kragel analyzed the data. Anne Botzung wrote the paper with comments from Kevin S. LaBar, Philip Kragel, and David C. Rubin.

\section{ACKNOWLEDGMENTS}

The authors wish to thank Jennifer Labrecque for her work in developing the basketball questionnaires and stimuli, the Duke Department of Athletics for providing the video footage, and William Heath for video editing. Supported by U.S. National Institutes of Health grants R01 AG023123 and 2 P01 NS041328.

decision-making. J. Neurosci. 27, 8161-8165.

Bartels,A., and Zeki, S. (2004a). Functional brain mapping during free viewing of natural scenes. Hum. Brain Mapp. 21, 75-85.

Bartels, A., and Zeki, S. (2004b). The chronoarchitecture of the human brain - natural viewing conditions reveal a time-based anatomy of the brain. Neuroimage 22, 419-433.

Bartels, A., and Zeki, S. (2005). The chronoarchitecture of the cerebral cortex. Philos. Trans. R. Soc. Lond., B, Biol. Sci. 360, 733-750.

Bell, A. J., and Sejnowski, T. J. (1995). An information-maximization approach to blind separation and 
blind deconvolution. Neural Comput. 7,1129-1159.

Blumenfeld, R. S., and Ranganath, C. (2007). Prefrontal cortex and longterm memory encoding: an integrative review of findings from neuropsychology and neuroimaging. Neuroscientist 13, 280-291.

Botzung, A., Denkova, E., Ciuciu, P., Scheiber, C., and Manning, L. (2008). The neural bases of the constructive nature of autobiographical memories studied with a self-paced fMRI design. Memory 16, 351-363.

Brown, R. (1986). Social Psychology, 2nd Edn. New York: The Free Press.

Buckner, R., Andrews-Hanna, J., and Schacter, D. L. (2008). The brain's default network: anatomy, function, and relevance to disease. Ann. N. Y. Acad. Sci. 1124, 1-38.

Cabeza, R., and St Jacques, P. (2007). Functional neuroimaging of autobiographical memory. Trends Cogn. Sci. 11, 219-227.

Calhoun, V. D., Adali, T., Pearlson, G. D., and Pekar, J. J. (2001). A method for making group inferences from functional MRI data using independent component analysis. Hum. Brain Mapp. 14, 140-151.

Calhoun, V. D., Adali, T., Pekar, J. J., and Pearlson, G. D. (2003). Latency (in)sensitive ICA. Group independent component analysis of fMRI data in the temporal frequency domain. Neuroimage 20, 1661-1669.

Calhoun, V.D., Liu, J., and Adali, T. (2009). A review of group ICA for fMRI data and ICA for joint inference of imaging, genetic, and ERP data. Neuroimage 45, 63-72.

Craig, A. D. (2002). How do you feel? Interoception: the sense of the physiological condition of the body. Nat. Rev. Neurosci. 3, 655-666.

Damoiseaux, J. S., Rombouts, S. A., Barkhof, F., Scheltens, P., Stam, C. J., Smith, S. M., and Beckmann, C. F. (2006). Consistent resting-state networks across healthy subjects. Proc. Natl. Acad. Sci. U.S.A. 103, 13848-13853.
Delgado, M. R. (2007). Reward-related responses in the human striatum. Ann. N. Y. Acad. Sci. 1104, 70-88.

Dolcos, F., and Denkova, E. (2008). Neural correlates of encoding emotional memories: a review of functional neuroimaging evidence. Cell Sci. Rev. 5, 78-122.

Dolcos, F., LaBar, K. S., and Cabeza, R. (2004). Dissociable effects of arousal and valence on prefrontal activity indexing emotional evaluation and subsequent memory: an event-related fMRI study. Neuroimage 23, 64-74.

Esposito, F., Bertolino, A., Scarabino, T., Latorre, V., Blasi, G., Popolizio, T., Tedeschi, G., Cirillo, S., Goebel, R., and Di Salle, F. (2006). Independent component model of the defaultmode brain function: assessing the impact of active thinking. Brain Res. Bull. 70, 263-269.

Fredrickson, B.L. (2001). The role of positive emotions in positive psychology. The broaden-and-build theory of positive emotions. Am. Psychol. 56, 218-226.

Golland, Y., Bentin, S., Gelbard, H., Benjamini, Y., Heller, R., Nir, Y., Hasson, U., and Malach, R. (2007). Extrinsic and intrinsic systems in the posterior cortex of the human brain revealed during natural sensory stimulation. Cereb. Cortex 17, 766-777.

Hasson, U., Furman, O., Clark, D., Dudai, Y., and Davachi, L. (2008). Enhanced intersubject correlations during movie viewing correlate with successful episodic encoding. Neuron $57,452-462$.

Jääskeläinen, I., Koskentalo, K., Balk, M. H., Autti, T., Kauramäki, J., Pomren, C., and Sams, M. (2008). Inter-subject synchronization of prefrontal cortex hemodynamic activity during natural viewing. Open. Neuroimag. J. 2, 14-19.

Kensinger,E.A., and Schacter,D.L. (2008). Neural processes supporting young and older adults' emotional memories. J. Cogn. Neurosci. 20, 1161-1173.

Kilpatrick, L., and Cahill, L. (2003). Amygdala modulation of parahippocampal and frontal regions during emotionally influenced memory storage. Neuroimage 20, 2091-2099.

LaBar, K. S., and Cabeza, R. (2006). Cognitive neuroscience of emotional memory. Nat. Rev. Neurosci. 7, 54-64.

LaBar, K. S., Gitelman, D. R., Parrish, T. B., and Mesulam, M. (1999). Neuroanatomic overlap of working memory and spatial attention networks: a functional MRI comparison within subjects. Neuroimage 10 695-704.

Li, Y. O., Adali, T., and Calhoun, V. D. (2007). Estimating the number of independent components for functional magnetic resonance imaging data. Hum. Brain Mapp. 28, 1251-1266.

Malinen, S., Hlushchuk, Y., and Hari, R. (2007). Towards natural stimulation in fMRI - issues of data analysis. Neuroimage 35, 131-139.

McIntosh, A. R., Bookstein, F. L., Haxby, J. V., and Grady, C. L. (1996). Spatial pattern analysis of functional brain images using partial least squares. Neuroimage 3, 143-157.

Mickley, K. R., and Kensinger,E.A. (2008). Emotional valence influences the neural correlates associated with remembering and knowing. Cogn. Affect. Behav. Neurosci. 8, 143-152.

Moran, J. M., Wig, G. S., Adams, R. B. Jr., Janata, P., and Kelley, W. M. (2004). Neural correlates of humor detection and appreciation. Neuroimage 21 , 1055-1060.

Ritchey, M., Dolcos, F., and Cabeza, R. (2008). Role of amygdala connectivity in the persistence of emotional memories over time: an event-related fMRI investigation. Cereb. Cortex 18 2494-2504.

Simons, J. S., and Spiers, H. J. (2003). Prefrontal and medial temporal lobe interactions in long-term memory. Nat. Rev. Neurosci. 4, 637-648.

Svoboda,E., McKinnon, M.C., and Levine, B. (2006). The functional neuroanatomy of autobiographical memory: a meta-analysis. Neuropsychologia 44, 2189-2208.
Van Overwalle, F. (2009). Social cognition and the brain: a meta-analysis. Hum. Brain Mapp. 30, 829-858.

Wann, D. L., and Branscombe, N. R. (1993).Sports fans: measuring degree of identification with their team. Int. J. Sport Psychol. 1-17.

Wann, D. L., Friedman, K., McHale, M., and Jaffe, A. (2003). The norelco sport fanatics survey: examining behaviors of sport fans. Psychol. Rep. 92, 930-936.

Warrington,E.(1984).RecognitionMemory Test. Windsor: NFER-Nelson.

Warrington, E. (1996). Topographical Recognition Memory Test. Hove, Sussex: Psychology Press.

Wolf, R. C., Sambataro, F., Vasic, N., Schönfeldt-Lecuona, C., Ecker, D., and Landwehrmeyer, B. (2008). Aberrant connectivity of lateral prefrontal networks in presymptomatic Huntington's disease. Exp. Neurol. 213, 137-44.

Zald, D. H. (2003). The human amygdala and the emotional evaluation of sensory stimuli. Brain Res. Rev. 41, $88-123$.

Conflict of Interest Statement: The authors declare that the research was conducted in the absence of any commercial or financial relationships that could be construed as a potential conflict of interest.

Received: 15 September 2009; paper pending published: 11 February 2010; accepted: 09 April 2010; published online: 18 May 2010.

Citation: Botzung A, LaBar KS, Kragel P, Miles A and Rubin DC (2010) Component neural systems for the creation of emotional memories during free viewing of a complex, real-world event. Front. Hum. Neurosit. 4:34. doi: 10.3389/fnhum.2010.00034 Copyright $\odot 2010$ Botzung, LaBar, Kragel, Miles and Rubin. This is an open-access article subject to an exclusive license agreement between the authors and the Frontiers Research Foundation, which permits unrestricted use, distribution, and reproduction in any medium, provided the original authors and source are credited. 